\title{
Which is the best predictor of thyroid cancer: thyrotropin, thyroglobulin or their ratio?
}

\author{
Pinar Yazici,, ${ }^{1}$ Mehmet Mihmanli, ${ }^{1}$ Emre Bozkurt, ${ }^{1}$ Feyza Yener Ozturk, ${ }^{2}$ Mehmet Uludag ${ }^{1}$ \\ ${ }^{1}$ General Surgery Clinic, ${ }^{2}$ Endocrinology and Metabolism Institute, Şişli Etfal Training and Research Hospital, Istanbul, \\ Turkey
}

\begin{abstract}
OBJECTIVE: Since documented incidence of thyroid cancer has been rising over time, in part due to incidental small papillary cancer, several studies have been carried out to investigate the role of possible serum markers of thyroid cancer prior to surgery. DESIGN: Prospective cohort study. AIM: To investigate the role of thyroglobulin (Tg), thyrotropin (TSH) and the TSH: Tg, Tg:TSH ratio in the preoperative diagnosis of thyroid cancer. PATIENTS AND METHODS: Between January 2014 and January 2015, 202 of 242 consecutive patients scheduled for surgical treatment for thyroid disorders at Şişli Etfal Training and Research Hospital, General Surgery Clinic were included in the study. Prospective data collection included demographics, thyroid function tests, Tg levels, fine needle aspiration biopsy (FNAB) results (208 FNABs in 187 patients), surgical procedures and final histopathological examinations. RESULTS: There were 134 patients with benign thyroid diseases and 68 patients with thyroid gland malignancy. Preoperative Tg level was significantly lower in the malignant group $(64 \mathrm{ng} / \mathrm{mL} \mathrm{vs.} 20 \mathrm{ng} / \mathrm{mL}$, $\mathbf{p}<\mathbf{0 . 0 0 1}$, respectively), whereas there was no difference in TSH between groups. TSH:Tg levels were found to be significantly higher in patients with malignant thyroid diseases $(0.24 \pm 1 \mathrm{vs}$. $0.87 \pm 3.4, p=0.024)$. Although univariate analysis showed that the TSH:Tg ratio was a predictor for thyroid malignancy (OR $0.001 ; 95 \% \mathrm{CI}, 0.01-0.125 ; p=0.007)$ in conjunction with FNAB, multivariate analysis failed to demonstrate any statistical significance for any factor except FNAB. CONCLUSION: Preoperative assessment of serum Tg and TSH levels appear not to be helpful in identifying patients with thyroid cancer. However, a higher preoperative serum TSH:Tg ratio may hint at an increased risk for thyroid carcinoma. Further studies are needed to elucidate the potential role of preoperative laboratory values of thyroid function.
\end{abstract}

Key words: Preoperative diagnosis, Thyroid cancer, Thyroglobulin, Thyrotropin

Address for correspondence:

Pinar Yazici, MD, Şişli Etfal Training and Research Hospital, Halaskargazi Cad. Etfal Sokak, 34371, Istanbul, Turkey;

Tel.: +90 (212) 373-5000, Fax: +90 21222407 72,

E-mail: drpinaryazici@gmail.com

Received: 18-12-2015, Accepted: 05-04-2016

\section{INTRODUCTION}

The increasing rate of incidental thyroid cancer poses a managerial dilemma in the preoperative diagnosis of thyroid nodules. Although fine needle aspiration/ biopsy (FNAB) is the most commonly used 
diagnostic technique for the preoperative evaluation of thyroid nodules, there remains the issue of gray-zone nodules that need further diagnostic investigation. To avoid an inappropriate surgical decision, additional preoperative markers have been suggested as being capable of providing prognostic and predictive value, with many as yet under investigation. During the last decade, interest has focused on the potential efficacy of thyroglobulin $(\mathrm{Tg})$ levels to identify preoperative malignancy in thyroid nodules, since it is an easily accessible and low-cost method.

The serum Tg level is believed to be a useful marker for the detection of well-differentiated thyroid cancer recurrence. However, its role as a predictor of thyroid malignancy in the preoperative workup of patients with thyroid nodules as yet remains controversial. Some studies have reported that in patients with indeterminate FNAB, Tg can be useful to detect thyroid malignancy in the preoperative period and for planning the surgical procedure. ${ }^{1,2}$ Likewise, in a retrospective analysis including patients with follicular neoplasm, Petric et al noted that in addition to age and presence of a solitary tumor, serum $\mathrm{Tg}$ concentration was also useful as an independent predictive marker to make the distinction between a benign and a malign follicular neoplasm. ${ }^{3}$

Among other preoperative markers for thyroid cancer, serum thyrotropin (TSH) levels are still under evaluation for the prediction of malignancy in thyroid nodules. Although TSH suppression treatment provides better disease-free survival in high-risk patients with differentiated thyroid cancer (DTC) ${ }^{4}$ some studies show no relationship between high TSH levels and thyroid malignancy. ${ }^{5}$ In contrast, in their recent study McLeod reported an association between high TSH levels and a higher stage of differentiated thyroid cancer. ${ }^{6}$ Furthermore, a correlation was found between TSH levels and extrathyroidal extension of thyroid cancer as well as neck node metastases. ${ }^{6,7}$

Not only indeterminate cytology but also certain other suspicious findings in thyroid FNAB are still a matter of discussion among endocrine surgeons. This debate is of great importance with respect to the determination of the most appropriate treatment procedures with, more specifically, the objective of reducing the number of unnecessary thyroid surgeries and the extend of the surgical procedure. Therefore, reliable predictive factors for malignancy are still warranted to avoid further interventions. ${ }^{3,8,9}$ This study aimed to investigate the efficacy of preoperative $\mathrm{Tg}$ and TSH levels for prediction of thyroid malignancy in patients undergoing thyroid surgery.

\section{PATIENTS AND METHODS}

Between January 2014 and January 2015, all patients who underwent thyroid surgery were included in the study. Those patients with a diagnosis of medullary thyroid cancer or with elevated antithyroglobulin antibody $(\mathrm{TgAb})$ and very elevated TSH were excluded, while patients with high-normal or slightly higher than normal TSH levels were included in the study. The study was approved by the Ethical Committee of Sisli Etfal Training and Research Hospital (491-92013) and informed consent was waived. Data were collected prospectively. Demographic features, type of thyroid disorder, any medication that can affect thyroid hormone levels, FNAB results, surgical procedures and histopathological examinations were recorded. In all patients, preoperative FT3, FT4, thyrotropin, Tg, $\mathrm{TgAb}$ and antithyroid peroxidase (TPO-Ab) levels were measured preoperatively. FNAB results were classified into five categories: nondiagnostic, benign, indeterminate, suspicious for malignancy and malignant. All patients were divided into two groups (benign group and malignant group) according to the final histopathological results.

\section{THYROID HORMONE MEASUREMENTS}

Plasma thyroid hormones (FT3, FT4, TSH) were analyzed using electrochemiluminescence (ECL) immunoassays. The normal values of thyroid hormones ranged between 2.7 and $5.7 \mathrm{pg} / \mathrm{mL}$ for FT3, between 0.7 and $1.7 \mathrm{ng} / \mathrm{dL}$ for FT4 and between 0.2 to $4.7 \mathrm{IU} /$ $\mathrm{mL}$ for $\mathrm{TSH}$.

$\mathrm{Tg}, \mathrm{TgAb}$ and TPOAb levels were measured using ECL assays (Elecsys, COBAS, e411, Roche Diagnostics $\mathrm{GmbH}$, Germany). The reference range for $\mathrm{Tg}$, $\mathrm{TgAb}$ and TPOAb were 1.4-78 ng/mL, 0-115 IU/mL, and $0-35 \mathrm{IU} / \mathrm{mL}$, respectively. 


\section{STATISTICAL ANALYSIS}

All patients were divided into the two groups: benign or malignant neoplasm of the thyroid gland (DTC); papillary thyroid carcinoma (PTC) or follicular thyroid carcinoma (FTC) according to the histopathological examinations. Numerical variables were expressed as mean \pm standard deviation (SD) or median (range) based on distribution pattern, while categorical variables were presented as absolute values and percentages. Differences between continuous and categorical variables were assessed by Student's $t$ test for normally distributed variables and the MannWhitney U test for non-normally distributed variables, and Fisher's exact test or the chi-square test, respectively. Univariate and multivariate analyses were carried out to identify variables associated with DTC. The receiver operating curve (ROC) analysis was performed to determine cut-offs of measured variables that were significant for predicting DTC. A P value less than 0.05 was considered statistically significant and all analyses were performed using SPSS, version 20 (Statistical Package for the Social Sciences Inc, Chicago, IL, USA).

\section{RESULTS}

There were 242 patients with a mean age of $48.2 \pm 11$ years who underwent thyroid surgery during the study period. Only 202 patients (169 female and 33 male), who met the inclusion criteria, were enrolled in the study. Of these patients, 134 had a final histopathological diagnosis of benign thyroid diseases, while 68 patients were confirmed with DTC. The malignant group included 62 PTCs (32 micropapillary carcinoma and 10 PTC with follicular variant), 5 FTC and one patient with metastatic renal cell carcinoma to the thyroid gland. Benign pathologies were composed of adenomatous nodular hyperplasia $(\mathrm{n}=74,55 \%$, concomitant lymphocytic thyroiditis: 22 and oncocytic changes: 15$)$, follicular adenoma $(n=23,17 \%)$ and others $(n=37,27 \%)$ (Figure 1).

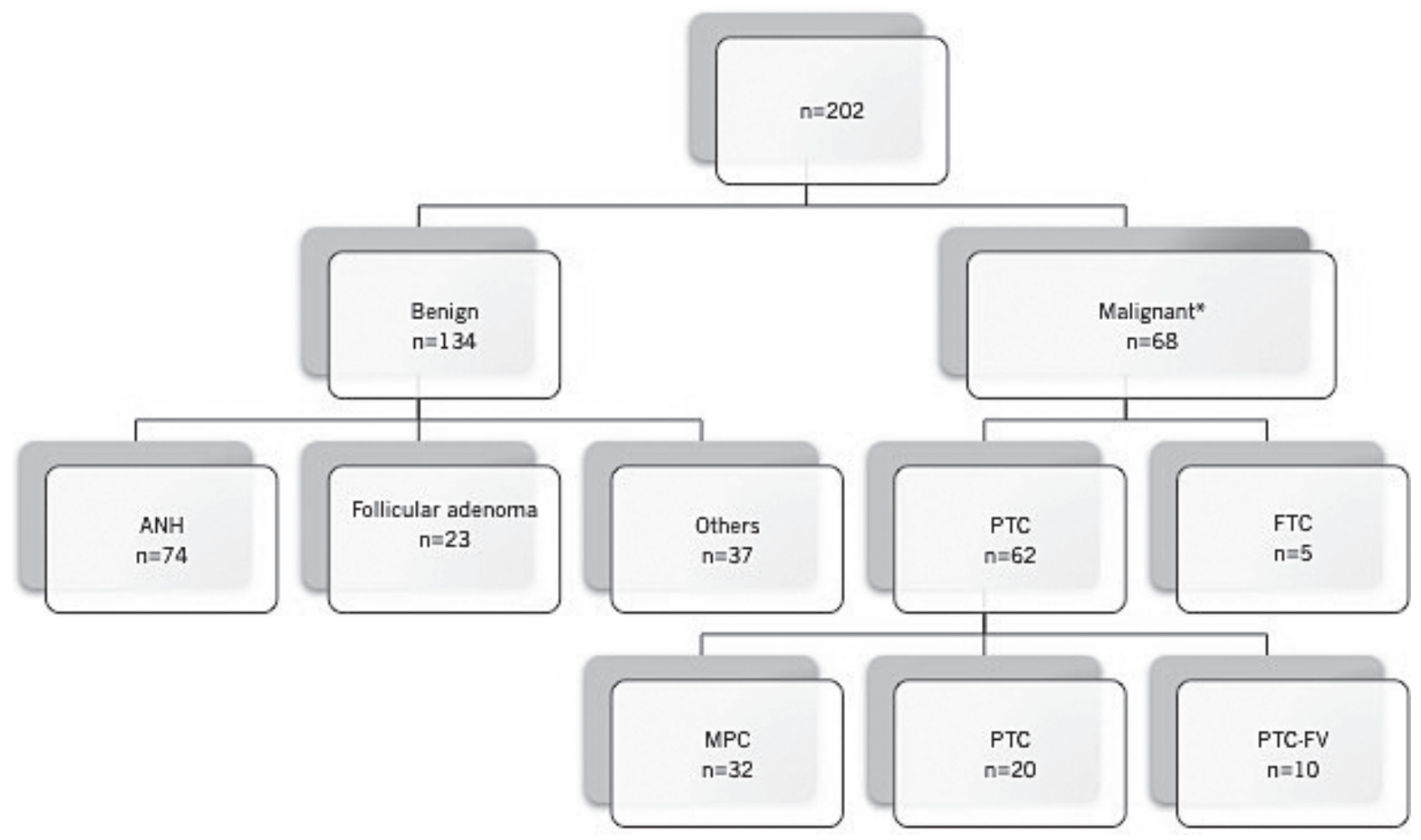

* One patient with metastatic renal cell carcinoma to the thyroid gland.

ANH: adenomatous nodular hyperplasia, PTC: papillary thyroid carcinoma, FTC: follicular thyroid carcinoma, MPC: micropapillary thyroid carcinoma, PTC: papillary thyroid carcinoma, FV: follicular variant.

Figure 1. Distribution of histopathological examination results of 202 patients 
Demographic and risk factors for thyroid cancer such as radiation exposure or family history were similar in both groups. Table 1 shows the types of thyroid disorders and surgical procedures in detail. Only four patients underwent completion thyroidectomy. Preoperative mean values of FT3 and FT4 results were in the normal range in the benign and malignant groups $(3.5 \pm 1.1 \mathrm{pg} / \mathrm{mL}$ and $3.6 \pm 1.2 \mathrm{pg} / \mathrm{mL}$, $1.2 \pm 0.4 \mathrm{ng} / \mathrm{dL}$ and $1.2 \pm 0.3 \mathrm{ng} / \mathrm{dL})$, respectively. Eight patients were under medical treatment for toxic nodular goiter and nine patients were taking medication for hypothyroidism. In both groups, mean anti-thyroid peroxidase (anti-TPO) levels were above the normal range $(47 \pm 9.5 \mathrm{IU} / \mathrm{mL}$ and $53 \pm 15 \mathrm{IU} / \mathrm{mL})$, whereas mean anti-Tg values were found to be within the normal range $(29 \pm 3 \mathrm{IU} / \mathrm{mL}$ and $41 \pm 12 \mathrm{IU} / \mathrm{mL})$. Mean levels of thyroid antibodies including anti-TPO and $\mathrm{TgAb}$ were similar between groups (Table 1).

Table 1. Comparison of demographic features and laboratory results between patients with benign and malignant thyroid disorders.

\begin{tabular}{|c|c|c|c|}
\hline & $\begin{array}{c}\text { Benign } \\
(\mathrm{n}=134,66 \%)\end{array}$ & $\begin{array}{c}\text { Malignant } \\
(\mathrm{n}=\mathbf{6 8}, \mathbf{3 3 \%})\end{array}$ & $\mathbf{p}$ \\
\hline \multicolumn{4}{|l|}{ Demographics } \\
\hline Age, years & $47 \pm 12$ & $49 \pm 12$ & 0.463 \\
\hline Gender (F/M) & $111 / 23$ & $57 / 11$ & 0.971 \\
\hline Preoperative drug use & 14 & 3 & 0.184 \\
\hline \multicolumn{4}{|l|}{ Laboratory results } \\
\hline TSH, IU/mL & $1.4 \pm 1.2,(1.07)$ & $1.9 \pm 2.5,(1.2)$ & 0.257 \\
\hline $\operatorname{Tg}(\mathrm{ng} / \mathrm{mL})^{\beta}$ & $64(0.25-1286)$ & $20(0.07-567)$ & $<0.001$ \\
\hline $\operatorname{Tg}($ normal / high, \%*) & $78 / 56,(41 \%)$ & $46 / 22,(32 \%)$ & 0.073 \\
\hline Anti-Tg (IU/mL) & $29 \pm 3.8$ & $41 \pm 12$ & 0.263 \\
\hline Anti-TPO (IU/mL) & $47 \pm 9$ & $53 \pm 15$ & 0.715 \\
\hline $\mathrm{TSH} / \mathrm{Tg}^{\beta}$ & $0.02(0.004-8.6)$ & $0.04(0.002-19)$ & 0.024 \\
\hline $\mathrm{Tg} / \mathrm{TSH}^{\beta}$ & $43(1-9100)$ & $17(1-5200)$ & 0.072 \\
\hline FNAB** (ND / B / I SM / M) & $8 / 105 / 17 / 4 / 2$ & $4 / 16 / 10 / 11 / 31$ & $12 / 121 / 27 / 15 / 33$ \\
\hline \multicolumn{4}{|l|}{ Type of thyroid disorders } \\
\hline Multi-nodular goiter & 103 & 62 & \\
\hline Solitary thyroid nodule & 26 & 5 & \\
\hline Toxic goiter (diffuse, nodular, multinodular) & 5 & 1 & \\
\hline \multicolumn{4}{|l|}{ Surgical procedures $(\mathrm{n}=210)^{\alpha}$} \\
\hline Lobectomy & $35(3)^{\&}$ & $4(1)^{8}$ & \\
\hline Total thyroidectomy & 102 & 65 & \\
\hline
\end{tabular}

Data are expressed as mean \pm standard deviation (SD) for normally distributed variables and median (range) for non-normally distributed variables.

Differences between continuous and categorical variables were assessed by Student's t test for normally distributed variables and the Mann-Whitney U test ${ }^{\beta}$ for non-normally distributed variables, and Fisher's exact test, respectively.

* Percentages show the frequency of higher Tg levels (above the normal range).

** A total of 208 FNAB results in 187 patients were evaluated. In the malignant group, 65 FNABs were performed in 62 patients, while in the benign group, 143 FNABs were performed in 125.

ND: non-diagnostic; B: benign; I: indeterminate; SM: suspicious for malignancy; M: malignant; Tg: thyroglobulin; TSH: thyroidstimulating hormone-thyrotropin; TPO-Ab: thyroperoxidase antibody; FNAB: fine needle aspiration biopsy.

\& completion thyroidectomy,

${ }^{\alpha}$ completion thyroidectomy (4), central neck dissection (2) and parathyroidectomy (2)

$\beta$ Mann-Whitney U test. 
The serum $T g$ level was found to be significantly higher in the benign group (64 ng/mL vs $20 \mathrm{ng} / \mathrm{mL}$, $\mathrm{p}<0.001$ ) (Table 1). Meanwhile, the rate of elevated $\mathrm{Tg}$ levels was $41 \%$ in the benign group, whereas it was $32 \%$ in the malignant group $(\mathrm{p}=0.073)$. TSH levels were observed to be similar $(1.4 \pm 1.2 \mathrm{IU} / \mathrm{mL}$ vs $1.9 \pm 2.5$ $\mathrm{IU} / \mathrm{mL}, \mathrm{p}=0.257)$. While the median Tg:TSH ratio was not statistically significant (43 vs $17, \mathrm{p}=0.07$ ), the median TSH:Tg ratio was significantly higher in patients with DTC ( 0.04 vs. $0.02, p=0.024)$. However, the ROC curve analysis failed to show a validated cut-off value which would allow preoperative diagnosis of thyroid cancer.

The results of the univariate and multivariate analyses are listed in Table 2. Univariate analysis showed that the TSH:Tg ratio was a predictor for thyroid malignancy (OR 0.001; 95\% CI, 0.01-0.125; $\mathrm{p}=0.007$ ) in combination with the FNAB results. When these parameters were included in a multivariate model, after adjustment for age and sex, it was no longer statistically significant.

Table 2. Univariate and multivariate analysis for thyroid cancer risk

\begin{tabular}{lcccc}
\hline & $\mathbf{p}$ & $\mathbf{O R}$ & $\mathbf{9 5 \%}$ & $\mathbf{C I}$ \\
\hline $\begin{array}{l}\text { Univariate regression } \\
\text { analysis }\end{array}$ & & & & \\
TSH, IU/mL & 0.889 & 0.000 & 0.000 & $\ldots$. \\
Tg, ng/mL & 0.745 & 1.000 & 0.998 & 1.001 \\
TSH:Tg & $\mathbf{0 . 0 0 7}$ & 0.001 & 0.000 & 0.125 \\
Tg:TSH & 0.184 & 1.183 & 0.923 & 1.517 \\
FNAB & $<\mathbf{0 . 0 0 1}$ & 30.004 & 8.033 & 112.064 \\
TPOAb, IU/mL & 0.685 & 0.999 & 0.995 & 1.003 \\
TgAb, IU/mL & 0.370 & 1.003 & 0.997 & 1.009 \\
Multivariate regression & & & & \\
analysis* & & & & \\
Age & 0.929 & 1.002 & 0.961 & 1.044 \\
Sex & 0.258 & 0.389 & 0.076 & 1.998 \\
TSH, IU/mL & 0.611 & 1.096 & 0.770 & 1.561 \\
Tg, ng/mL & 0.525 & 0.999 & 0.996 & 1.002 \\
TSH:Tg & 0.762 & 0.982 & 0.872 & 1.106 \\
FNAB & $<\mathbf{0 . 0 0 1}$ & 25.034 & 4.907 & 127.729 \\
\hline
\end{tabular}

*after adjustment for sex and age

TSH: thyrotropin, Tg: thyroglobulin, FNAB: fine needle aspiration biopsy, TPO-Ab: anti-thyroperoxidase antibody, TgAb: Thyroglobulin antibody.
With regard to the preoperative FNAB results, nondiagnostic or indeterminate pathology was detected in 42 patients. Of these, in 14 patients (33\%) malignant thyroid disorders were confirmed following the final histopathological examination. Those patients with an FNAB that resulted in a suspicious for malignancy or a malignant thyroid nodule $(\mathrm{n}=48)$ had both higher TSH levels $(2.0 \mathrm{IU} / \mathrm{mL}$ vs $1.4 \mathrm{IU} / \mathrm{mL}, \mathrm{p}=0.08)$ and a higher thyrotropin:thyroglobulin ratio ( 0.9 vs 0.3 , $\mathrm{p}=0.093$ ). The rate of malignant thyroid disorders in these patients was $87 \%$.

\section{DISCUSSION}

Although there have been several studies investigating the association between preoperative $\mathrm{Tg}$ and TSH levels and malignant thyroid disorders, neither of them has so far been demonstrated as having sufficient accuracy to be used as a screening test. ${ }^{10}$ The present study confirms that the simultaneously increased levels of both Tg and TSH are not useful in predicting thyroid malignancy in the preoperative period. By contrast, Tg levels were significantly lower in patients with DTC. In addition, recent studies have demonstrated an inverse or direct relationship between the DTC and $\mathrm{Tg}$ :TSH ratio ${ }^{11}$ or the TSH:Tg ratio. ${ }^{12}$ In our study, we consistently observed a direct correlation between the TSH:Tg ratio and DTC, whereas no relationship was found with the Tg:TSH ratio.

A number of diagnostic procedures are needed to prevent unnecessary surgery. While ultrasonographyguided FNAB is still the most widely used diagnostic tool for thyroid cancer, ${ }^{13-15}$ one of the reasons for inconclusive results is its low-sensitivity, particularly for certain disorders. ${ }^{15-17}$ Apart from the fact that FNAB results not infrequently include indeterminate cytology and suspicious findings for malignant thyroid nodules, FNA biopsy needs a highly qualified cytopathologist who will guide the clinician to consider other additional diagnostic methods. There have been several studies showing the benefits of measuring Tg levels in patients with indeterminate FNAB cytology. ${ }^{1}$ In our study, preoperative Tg levels were not significantly higher in this group of patients. Another challenge related to FNAB indeterminate specimens is that most of the final pathological results for these nodules reveal benign findings, as shown in 
our study. ${ }^{18-20}$ Histopathological results confirmed malignancy at a rate of $33 \%$ for our series. Another factor associated with false negative FNAB and subsequent unnecessary surgery is incidental microcarcinomas, with a rate reaching $40 \%{ }^{21}$ In this study, the rate of micropapillary thyroid carcinoma was almost 50\%.

Given the aforementioned limitations of FNAB, several other potentially predictive factors have been studied for the detection of malignancy risk in thyroid nodules. Particularly molecular markers, such as BRAF mutations in a FNAB specimen, are expected to have a significant impact on cancer prognostication and are increasingly being resorted to for inconclusive cases. ${ }^{22}$ Other molecular markers, including galectin-3 and cytokeratin-19, have been also been used to improve diagnostic accuracy for indeterminate nodules..$^{23-25}$ Nevertheless, concerns about accessibility and costeffectiveness still impede the widespread use of these markers in the clinical setting. Several articles have assessed the role of preoperative serum hormones, antibodies and a number of proteins for predicting malignancy in thyroid nodules. However, the diagnostic role of these markers (thyrotropin, TgAb, etc.) and $\mathrm{Tg}$ remains debatable.

Serum Tg level is not specific for thyroid cancer and can also be detected at elevated levels in several proliferative thyroid disorders. ${ }^{1,26}$ Neither the ATA nor the AACE/ETA/AME guidelines recommend the assessment of serum $\mathrm{Tg}$ in the diagnosis of thyroid nodules. ${ }^{27}$ Likewise, several studies have shown preoperative $\mathrm{Tg}$ measurement to be useless because it is too weak to distinguish between malignant nodules and benign, ${ }^{1,26,28,29}$ whereas others have noted its potential to predict thyroid malignancy in the preoperative period. ${ }^{2,3}$ Conversely, some other series, including the present study, have reported lower Tg levels in patients with thyroid malignancy compared to those with benign disorders. ${ }^{11,29}$

Thyrotropin is another popular biomarker considered to be a predictor for differentiated thyroid cancer. ${ }^{30,31}$ However, although it was demonstrated that DTCs express TSH receptors, ${ }^{32}$ the issue is still under discussion. Some studies showed an increased risk of malignancy in a thyroid nodule with high serum TSH, $, 7,30,31$ whereas others reported no relationship. ${ }^{10,33-35}$ In a meta-analysis including over 5000 patients with thyroid cancer, McLeod et al showed that the risk of thyroid cancer was two-fold higher in patients with increased TSH levels. ${ }^{6}$ The most recent version of the ATA guidelines also highlight the association between higher serum TSH levels and increased risk of malignancy in a thyroid nodule. ${ }^{36}$ There are also some studies showing a direct correlation between higher serum TSH levels and higher tumor stage..$^{31,37}$

Currently, there is increasing interest in the development of novel, easily applied markers that have the potential to enhance our ability to predict thyroid malignancy in the preoperative period. Today, preoperative serum markers (Tg, TSH and thyroid antibodies) are attracting growing interest among clinicians because they are fairly accessible tests, faster and low-cost by contrast with other methods. However, they have demonstrated suboptimal accuracy in discriminating malignant from benign nodules. Recently, Wang et al investigated the role of the thyrotropin:thyroglobulin ratio to predict malignancy and found that it was significantly higher in patients with DTC, ${ }^{12}$ whereas Cohen et al showed a significantly lower Tg:TSH ratio with a cut-off value of 2.7 in patients with malign disorders. ${ }^{11}$ With regard to our results, TSH:Tg was significantly higher in the malignant group, whereas Tg:TSH was found to be lower in the malignant group, showing no statistically significant difference. Although TSH was not statistically significant between groups, the mean level of TSH was higher in the malignant group. This means that higher TSH levels and concomitant lower (normal or high-normal) $\mathrm{Tg}$ levels should alert the clinician to the possibility of thyroid cancer.

Our study has several strengths, including its prospective design, the large number of patients with DTC and the exclusion of those with high TgAb and very elevated TSH levels requiring higher doses of antithyroid medication. Limitations included lack of data on nodule features from the ultrasonographic findings and the tumor diameter, which can have a correlation with these parameters, were not recorded.

\section{CONCLUSIONS}

Despite a growing amount of information regarding preoperative markers for the prediction of thyroid 
cancer preoperatively, an increased level of serum $\mathrm{Tg}$ or TSH appear w not to be useful to predict malignant thyroid disorders. However, TSH:Tg ratio was found to be significantly increased in patients with thyroid cancer but it was not shown to be an independent predictor. Large-scale prospective studies or meta-analyses will yield more conclusive evidence to clarify this issue.

\section{ETHICS COMMITTEE APPROVAL}

Ethics committee approval was obtained for this study from the Local Ethical Committee of Şişli Etfal Training and Research Hospital.

\section{CONFLICT OF INTEREST}

The authors do not have any conflict of interest.

\section{REFERENCES}

1. Lee EK, Chung KW, Min HS, et al, 2012 Preoperative serum thyroglobulin as a useful predictive marker to differentiate follicular thyroid cancer from benign nodules in indeterminate nodules. Korean Med Sci 27: 1014-1018.

2. Sands N, Karls S, Rivera J, et al, 2010 Preoperative serum thyroglobulin as an adjunct to fine-needle aspiration in predicting well-differentiated thyroid cancer. J Otolaryngol Head Neck Surg 39: 669-673.

3. Petric R, Perhavec A, Gazic B, Besic N, 2012 Preoperative serum thyroglobulin concentration is an independent predictive factor of malignancy in follicular neoplasms of the thyroid gland. J Surg Oncol 105: 351-356.

4. Rago T, Fiore E, Scutari M, et al, 2010 Male sex, single nodularity, and young age are associated with the risk of finding a papillary thyroid cancer on fine needle aspiration cytology in a large series of patients with nodular thyroid disease. Eur J Endocrinol 162: 763-770.

5. Sohn SY, Kim HJ, Jang HW, Kim SW, Chung JH, 2014 Lack of association between high serum thyroidstimulating hormone level and risk of papillary thyroid microcarcinomas. Head Neck 36: 43-46.

6. McLeod DS, Cooper DS, Ladenson PW, et al, 2014 Prognosis of differentiated thyroid cancer in relation to serum thyrotropin and thyroglobulin antibody status at time of diagnosis. Thyroid 24: 35-42.

7. Haymart MR, Glinberg SL, Liu J, Sippel RS, Jaume JC, Chen H, 2009 Higher serum TSH in thyroid cancer patients occurs independent of age and correlates with extrathyroidal extension. Clin Endocrinol (Oxf)71: 434-439.

8. Hocevar M, Auersperg M, 1998 Role of serum thy- roglobulin in the pre- operative evaluation of follicular thyroid tumors. Eur J Surg Oncol 24: 553-557.

9. Strazisar B, Petric R, Sesek M, Zgajnar J, Hocevar M, Besic N, 2010 Predictive factors of carcinoma in 279 patients with Hürthle cell neoplasm of the thyroid gland. J Surg Oncol 101: 582-586.

10. Rinaldi S, Plummer M, Biessy C, et al, 2014 Thyroidstimulating hormone, thyroglobulin, and thyroid hormones and risk of differentiated thyroid carcinoma: the EPIC study. J Natl Cancer Inst 106: Doi: 10.1093/ jnci/dju097

11. Cohen N, Pakdaman MN, Rochon L, Tamilia M, Richard J Payne MD, 2009 Tg:TSH Ratio as a Tumor Marker for Thyroid Cancer. Otolaryngol Head Neck Surg 141: 165

12. Wang L, Li H, Yang Z, Guo Z, Zhang Q, 2015 Preoperative serum thyrotropin to thyroglobulin ratio is effective for thyroid nodule evaluation in euthyroid patients. Otolaryngol Head Neck Surg 2015: pii: 0194599815579877.

13. Gharib H, Goellner JR, 1993 Fine-needle aspiration biopsy of the thyroid: an appraisal. Ann Intern Med 118: 282-289.

14. Frates MC, Benson CB, Charboneau JW, et al, 2005 Management of thyroid nodules detected at US: Society of Radiologists in Ultrasound Consensus Conference statement. Radiology 237: 794-800.

15. Miller B, Burkey S, Lindberg G, Snyder WH 3rd, Nwariaku FE, 2004 Prevalence of malignancy within cytologically indeterminate thyroid nodules. Am J Surg 188: 459-462.

16. Haigh PI, 2002 Follicular thyroid carcinoma. Current treatment options in oncology 3: 349-354.

17. Yeh MW, Demircan O, Ituare P, Clark OH, 2004 False negative fine needle aspiration cytology results delay treatment and adversely affect outcome in patients with thyroid carcinoma. Thyroid 14: 207-215.

18. Sherman SI, 2003 Thyroid carcinoma. Lancet 361: 501-511.

19. Führer D, 2001 A nuclear receptor in thyroid malignancy: is PAX8/PPAR the holly grail of follicular thyroid cancer. Eur J Endocrinol 144: 453-456.

20. DeMay RM, 2000 Follicular lesions of the thyroid. W(h)ither follicular carcinoma?. Am J Clin Pathol 114: 681-683.

21. Gul K, Ozdemir D, Ersoy R, et al, 2009 Comparison of Papillary Thyroid Microcarcinoma and Carcinoma. Turk J Endocrinol Metab 13: 47-51.

22. Xing M, 2005 BRAF mutation in thyroid cancer. Endocr Relat Cancer 12: 245-262.

23. Trimboli P, Guidobaldi L, Amendola S, et al, 2015 Galectin-3 and HBME-1 improve the accuracy of core biopsy in indeterminate thyroid nodules. Endocrine 52: 39-45.

24. Bartolazzi A, D’Alessandria C, Parisella MG, et al, 2008 Thyroid cancer imaging in vivo by targeting 
the anti-apoptotic molecule galectin-3. PLoS One 2008; 3: e3768.

25. Cui W, Sang W, Zheng S, Ma Y, Liu X, Zhang W, 2012 Usefulness of cytokeratin-19, galectin-3, and Hector Battifora mesothelial-1 in the diagnosis of benign and malignant thyroid nodules. Clin Lab 58: 673-680.

26. Cooper DS, Doherty GM, Haugen BR, et al, 2009 Revised American Thyroid Association management guidelines for patients with thyroid nodules and differentiated thyroid cancer. Thyroid 19: 1167-1214.

27. Gharib H, Papini E, Paschke R, et al, AACE/AME/ ETA Task Force on Thyroid Nodules, 2010 American Association of Clinical Endocrinologists, Associazione Medici Endocrinologi, and European Thyroid Association medical guidelines for clinical practice for the diagnosis and management of thyroid nodules. Endocr Pract 16: Suppl 1: 1-43.

28. Suh I, Vriens MR, Guerrero MA, et al, 2010 Serum thyroglobulin is a poor diagnostic biomarker of malignancy in follicular and Hurthle-cell neoplasms of the thyroid. Am J Surg 200: 41-46.

29. Yalçin S, Ulger BV, Parlak O, Ucar AE, Sarikaya SM, Ozer M, 2011 The role of preoperative serum thyroglobulin and thyroid auto-antibody levels before histopathological diagnosis of thyroid cancers. Turk J Med Sci 41: 487-493.

30. Boelaert K, Horacek J, Holder RL, Watkinson JC, Sheppard MC, Franklyn JA, 2006 Serum thyrotropin concentration as a novel predictor of malignancy in thyroid nodules investigated by fine-needle aspiration.
J Clin Endocrinol Metab 91: 4295-4301.

31. Haymart MR, Repplinger DJ, Leverson GE, et al, 2008 Higher serum thyroid stimulating hormone level in thyroid nodule patients is associated with greater risks of differentiated thyroid cancer and advanced tumor stage. J Clin Endocrinol Metab 93: 809-814.

32. Jameson JL, Weetman AP, 2001, In Thyroid Cancer. Harrison's Principles of Internal Medicine, Editors: Braunwald E, Fauci AS, Kasper DL, Hauser SL, Longo DL, Jameson JL (eds) S: 2079-2081.

33. Negro R, Valcavi R, Riganti F, et al, 2013 Thyrotropin values in patients with micropapillary thyroid cancer versus benign nodular disease. Endocr Pract 19: 651-655.

34. Kim HS, Lee SJ, Park JK, 2011 Association between serum thyroid stimulating hormone level and papillary thyroid microcarcinoma in Korean euthyroid patients. Endocrinol Metab 26: 297-302.

35. Tuna MM, Basaran MN, Karakilic E, et al, 2014 Diagnostic and Prognostic Value of TSH Levels in Differentiated Thyroid Cancers. Turk Jem 1: 1-4.

36. Haugen BR Md, Alexander EK, Bible KC, et al, 2015 American thyroid association management guidelines for adult patients with thyroid nodules and differentiated thyroid cancer. Thyroid 26: 1-133.

37. Fiore E, Rago T, Provenzale MA, et al, 2009 Lower levels of TSH are associated to a lower risk of papillary thyroid cancer in patients with thyroid nodular disease: thyroid autonomy may play a protective role. Endocr Relat Cancer 16: 1251-1260. 